\title{
Some concepts related to generalized Kuratowski conver- gence via ideal
}

\author{
Vakeel A. Khan ${ }^{\mathrm{a}, *}$, Ayhan Esi ${ }^{\mathrm{b}}$, Mobeen Ahmad $^{\mathrm{a}}$ \\ ${ }^{a}$ Department of Mathematics, Aligarh Muslim University, Aligarh 202002, India. \\ ${ }^{b}$ Department of Basic Engineering Sciences, Engineering Faculty, Malatya Turgut Ozal University, Malatya 44100, Turkey.
}

\begin{abstract}
The present article aims to study and extend the concepts of Kuratowski ideal convergence of sequence of closed sets in the framework of intuitionistic fuzzy metric spaces and obtain several properties for Kuratowski ideal convergent of sequence of closed sets.
\end{abstract}

Keywords: Ideal, filter, I-outer point, I-inner limit point, ideal convergence, Kuratowski I-convergence, intuitionistic fuzzy metric spaces.

2020 MSC: 40A35, 49J53, 54C60.

(C)2022 All rights reserved.

\section{Introduction and Preliminaries}

The notion of convergence of sequences of points has been extended by several researchers to the convergent of sequences of sets. Set convergence determined as the proportionality of outer limits and inner limits. In 1902, Painleve studied the outer limits and inner limits for the sequence of sets. This convergence has been fashionable by Kuratowski in his well-known book Topologie [12] and hence, frequently said to be Kuratowski convergence of sequences of sets. Fast [3] initiated the concept of statistical convergence. Later on, it was examined from the sequence viewpoint and linked it with the summability theory by Fridy[4, 5]. Fridy and Orhan [6] examined statistical limit superior and limit inferior. Moreover, Kostyrko et al. [11] generalized statistical convergence using the structure of the ideal I as a subset of set $\mathbb{N}$ of positive integers. Furthermore, it analyzed by Salat et al. [17], Khan et al. [9, 10], and many others.

Zadeh [21] initiated the theory of fuzzy sets in 1965. Atanassov [1] introduced the notion of intuitionistic fuzzy sets which was a generalization of fuzzy sets. The intuitionistic fuzzy theory has emerged as the most active area of research in many branches of mathematics and engineering. One of the most important problems in intuitionistic fuzzy topology is to obtain an appropriate concept of intuitionistic fuzzy metric space. George and Veeramani [7, 8] established the fuzzy metric spaces, and Park [15] further analyzed the intuitionistic fuzzy metric spaces. In continuation of the theory, Saadati et al. [16]

\footnotetext{
*Corresponding author

Email addresses: vakhanmaths@gmail.com (Vakeel A. Khan), aesi23@hotmail.com (Ayhan Esi), mobeenahmad88@gmail.com (Mobeen Ahmad)
}

doi: 10.22436/jnsa.015.02.01

Received: 2021-05-14 Revised: 2021-05-26 Accepted: 2021-07-12 
extended intuitionistic fuzzy metric spaces to intuitionistic fuzzy topological spaces. Later on, Mursaleen et al. $[13,14]$ studied statistical convergence and ideal convergence of double sequences in intuitionistic fuzzy normed spaces.

Definition 1.1 ([11]). A family of subsets $I \subset 2^{\mathbb{N}}$ is known as an ideal if and only if $\emptyset \in \mathrm{I}$, for each $\mathrm{U}, \mathrm{V} \in \mathrm{I}$ we have $\mathrm{U} \cup \mathrm{V} \in \mathrm{I}$ and for each $\mathrm{U} \in \mathrm{I}$ and $\mathrm{V} \subseteq \mathrm{U}$, we have $\mathrm{V} \in \mathrm{I}$.

Definition 1.2 ([11]). A non-void family of sets $\mathcal{F} \subset 2^{\mathbb{N}}$ is known as a filter on $\mathbb{N}$ if and only if $\emptyset \notin \mathcal{F}$, for $\mathrm{U}, \mathrm{V} \in \mathcal{F}$, we have $\mathrm{U} \cap \mathrm{V} \in \mathcal{F}$ and for each $\mathrm{U} \in \mathcal{F}$ and $\mathrm{U} \subseteq \mathrm{V}$, we have $\mathrm{V} \in \mathcal{F}$. An ideal $\mathrm{I}$ is said to be non-trivial if $I \neq 2^{\mathbb{N}}$. A non-trivial ideal I is said to be admissible if $\{\{n\}: n \in \mathbb{N}\} \subset I$.

Proposition 1.3 ([11]). For each ideal $\mathrm{I}$, there is a filter $\mathcal{F}(\mathcal{J})$ associated with $\mathrm{I}$ defined as $\mathcal{F}(\mathrm{I})=\left\{\mathrm{U} \subseteq \mathbb{N}: \mathrm{U}^{\mathrm{c}} \in \mathrm{I}\right\}$.

Lemma 1.4 ([11]). $\mathrm{K} \in \mathcal{F}(\mathrm{I})$ and $\mathrm{K}_{1} \subseteq \mathbb{N}$. If $\mathrm{K}_{1} \notin \mathrm{I}$, then $\mathrm{K}_{1} \cap \mathrm{K} \notin \mathrm{I}$.

Definition 1.5 ([19]). A sequence of sets $\left(E_{k}\right)$ is called I-monotonic increasing, if there exists a subset $P=\left\{k_{1}<k_{2}<k_{2}<\cdots\right\} \in \mathcal{F}(I)$ such that $E_{k_{i}} \subseteq E_{k_{i+1}}$ for every $i \in \mathbb{N}$. Similarly, sequence of sets $\left(E_{k}\right)$ is called I-monotonic decreasing, if there exist a subset $P=\left\{k_{1}<k_{2}<k_{2}<\cdots\right\} \in \mathcal{F}(I)$ such that $E_{k_{i}} \supseteq E_{k_{i+1}}$ for every $i \in \mathbb{N}$.

Definition 1.6. Let $(X, d)$ be a metric space. The open ball with radius $\varepsilon>0$ and center $X$ in $X$ is defined as $\mathrm{B}(\mathrm{x}, \varepsilon)=\{z \in \mathrm{X}: \mathrm{d}(\mathrm{x}, z)<\varepsilon\}$. tions:

Recalling the fundamental properties of Kuratowski convergence, we use the undermentioned nota-

$Q:=\{\mathrm{K} \subseteq \mathbb{N}: \mathbb{N} \backslash \mathrm{K}$ finite $\}:=\left\{\right.$ subsequences of $\mathbb{N}$ containing all $\mathrm{n}$ beyond some $\left.\mathrm{n}_{0}\right\} ;$

$Q^{\sharp}:=\{K \subseteq \mathbb{N}: K$ infinite $\}=\{$ all subsequences of $\mathbb{N}\}$.

Denote $\lim _{k \rightarrow \infty}$ when $k$ tends to infinity as usual in $\mathbb{N}$. On the other hand $\lim _{k \in K}$ in this case of convergence of a subsequence referred by an index set $K$ in $Q^{\sharp}$.

Talo and Sever [19] extended Kuratowski statistical convergence of the sequence of closed sets to Kuratowski I-convergence using the concept of Kuratowski [12] and Talo et al. [20] as follows.

Definition $1.7([19])$. Let $\left(E_{k}\right)$ be a sequence of closed subsets of $X$. Then I-outer limit and I-inner limit are defined by

$$
\mathrm{I}-\limsup _{\mathrm{k} \rightarrow \infty} \mathrm{E}_{\mathrm{k}}:=\left\{x \mid \forall \varepsilon>0, \exists \mathrm{K} \in \mathcal{Q}_{\mathcal{J}}^{\sharp}, \forall \mathrm{k} \in \mathrm{K}: \mathrm{E}_{\mathrm{k}} \cap \mathrm{B}(x, \varepsilon) \neq \emptyset\right\}
$$

and

$$
\mathrm{I}-\liminf _{\mathrm{k} \rightarrow \infty} \mathrm{E}_{\mathrm{k}}:=\left\{x \mid \forall \varepsilon>0, \exists \mathrm{K} \in \mathcal{Q}_{\mathcal{J}}, \forall \mathrm{k} \in \mathrm{K}: \mathrm{E}_{\mathrm{k}} \cap \mathrm{B}(x, \varepsilon) \neq \emptyset\right\},
$$

respectively, where, $Q^{\sharp}:=\{\mathrm{K} \subseteq \mathbb{N}: \mathrm{K} \notin \mathrm{I}\}$ and $Q_{\mathrm{I}}:=\{\mathrm{K} \subset \mathbb{N}: \mathbb{N} \backslash \mathrm{K} \in \mathrm{I}\}=\mathcal{F}(\mathrm{I})$.

In a sequence of sets $\left(E_{k}\right)$, I- limit exists if its I-inner limit and I-outer limit are equal. In this case, the sequence of sets is said to be Kuratowski I-convergent and it is noted as:

$$
I-\liminf _{k \rightarrow \infty} E_{k}=I-\limsup _{k \rightarrow \infty} E_{k}=I-\lim _{k \rightarrow \infty} E_{k} .
$$

Definition 1.8 ([15]). Let $X$ be a non-void set, $*$ is a continuous t-norm, $\diamond$ is a continuous $\mathrm{t}$-conorm and $M, N$ are fuzzy sets on $X^{2} \times(0, \infty)$. Then the five-tuple $(X, M, N, *, \diamond)$ is said to be an intuitionistic fuzzy metric space (for short, IFMS) if it fulfilling the subsequent conditions for all $s, t>0$ and for every $y, z, w \in X$

(a) $M(y, z, t)+N(y, z, t) \leqslant 1$;

(b) $M(y, z, t)>0$;

(c) $M(y, z, t)=1$ if and only if $y=z$; 
(d) $M(y, z, t)=M(z, y, t)$;

(e) $M(y, z, t) * M(z, w, s) \leqslant M(y, w, t+s)$;

(f) $M(y, z,):.(0, \infty) \rightarrow[0,1]$ is continuous;

(g) $\mathrm{N}(\mathrm{y}, z, \mathrm{t})<1$;

(h) $N(y, z, t)=0$ if and only if $y=z$;

(i) $\mathrm{N}(\mathrm{y}, z, \mathrm{t})=\mathrm{N}(z, \mathrm{y}, \mathrm{t})$;

(j) $\mathrm{N}(\mathrm{y}, z, \mathrm{t}) \diamond \mathrm{N}(z, w, \mathrm{~s}) \geqslant \mathrm{N}(\mathrm{y}, w, \mathrm{t}+\mathrm{s})$;

(k) $\mathrm{N}(y, z,):.(0, \infty) \rightarrow[0,1]$ is continuous.

In this case, $(M, N)$ is said to be an intuitionistic fuzzy metric.

Example 1.9 ([15]). Let $(X, d)$ be a metric space. Define $a \diamond b=\min (a+b, 1)$ and $a * b=a b$ for all $a, b \in[0,1]$ and let $M_{d}$ and $N_{d}$ be fuzzy sets on $X^{2} \times(0, \infty)$ defined as

$$
M_{d}(y, z, t)=\frac{p t^{n}}{p t^{n}+m d(y, z)}, \quad N_{d}(y, z, t)=\frac{d(y, z)}{q t^{n}+m d(y, z)}
$$

for all $m, n, p, q \in R^{+}$. Then $(X, M, N, *, \diamond)$ is an IFMS.

Definition $1.10([15])$. Let $(X, M, N, *, \diamond)$ be an IFMS. For $t>0$ and the radius $\varepsilon \in(0,1)$ with center $x \in X$, the open ball $\mathcal{B}_{\chi}(\varepsilon, t)$ is defined by

$$
\mathcal{B}_{x}(\varepsilon, t)=\{y \in X: M(x, y, t)>1-\varepsilon, N(x, y, t)<\varepsilon\} .
$$

Definition 1.11. Let $(X, M, N, *, \diamond)$ be an IFMS. An element $\xi \in X$ is called I- cluster point of sequence $x=\left(x_{k}\right)$ if

$$
\left\{k \in \mathbb{N}: M\left(\xi, x_{k}, t\right)>1-\varepsilon \text { and } N\left(\xi, x_{k}, t\right)<\varepsilon\right\} \notin I,
$$

for each $\varepsilon>0$ and $t>0$. I $\left(\Gamma_{\chi}\right)$ denotes the set of all I-cluster points of $x$.

Definition $1.12([16])$. Let $(X, M, N, *, \diamond)$ be an IFMS and $A$ be a non-void subset of $X$. For all $t>0$ and $x \in X$, define

$$
M(x, A, t)=\sup \{M(x, y, t): y \in A\}
$$

and

$$
N(x, A, t)=\inf \{N(x, y, t): y \in A\},
$$

where $M(x, A, t)$ and $N(x, A, t)$ are the degree of closeness and the degree of non-closeness of $x$ to $A$ at $t$, respectively.

The notion of I-limit superior and I-limit inferior were proposed by Demirci [2] and Sen [18] extended it into intuitionistic fuzzy normed spaces. Now, we discuss I-limit superior and inferior in IFMS as follows. Suppose $x=\left(x_{k}\right)$ be a real sequence and I be an admissible ideal;

$$
I_{(M, N)}-\limsup _{k \rightarrow \infty} x:=\left\{\begin{array}{ll}
\sup _{B_{x}^{(M, N)},} & B_{x}^{(M, N)} \neq \phi, \\
0, & B_{x}^{(M, N)}=\phi,
\end{array} \quad I_{(M, N)}-\liminf _{k \rightarrow \infty} x:= \begin{cases}\inf A_{x}^{(M, N)}, & A_{x}^{(M, N)} \neq \phi, \\
1, & A_{x}^{(M, N)}=\phi,\end{cases}\right.
$$

where,

$$
\begin{aligned}
& B_{x}^{(M, N)}=\left\{b \in(0,1):\left\{k \in \mathbb{N}: M\left(x_{k}, y, t\right)<1-b \text { or } N\left(x_{k}, y, t\right)>b\right\} \notin I\right\}, \\
& A_{x}^{(M, N)}=\left\{a \in(0,1):\left\{k \in \mathbb{N}: M\left(x_{k}, y, t\right)>1-a \text { or } N\left(x_{k}, y, t\right)<a\right\} \notin I\right\} .
\end{aligned}
$$

Proposition 1.13. If $\mathrm{I}_{(\mathrm{M}, \mathrm{N})}-\lim \sup \mathrm{x}=\mathrm{b}$ is finite, then for all $\mathrm{t}>0$ and for every $\varepsilon>0$,

$$
\begin{aligned}
& \left\{k \in \mathbb{N}: M\left(x_{k}, y, t\right)<1-b+\varepsilon \text { or } N\left(x_{k}, y, t\right)>b-\varepsilon\right\} \notin I, \\
& \left\{k \in \mathbb{N}: M\left(x_{k}, y, t\right)<1-b-\varepsilon \text { or } N\left(x_{k}, y, t\right)>b+\varepsilon\right\} \in I .
\end{aligned}
$$

Conversely if (1.3) and (1.4) hold for every $\varepsilon>0$ and $\mathrm{t}>0$, then $\mathrm{b}=\mathrm{I}_{(\mathrm{M}, \mathrm{N})}-\lim \sup \mathrm{x}$. 
Proposition 1.14. If $\mathrm{a}=\mathrm{I}_{(\mathrm{M}, \mathrm{N})}-\lim \inf \mathrm{x}$ is finite, then for all $\mathrm{t}>0$ and for every $\varepsilon>0$,

$$
\begin{aligned}
& \left\{k \in \mathbb{N}: M\left(x_{k}, y, t\right)>1-a-\varepsilon \text { or } N\left(x_{k}, y, t\right)<a+\varepsilon\right\} \notin I \\
& \left\{k \in \mathbb{N}: M\left(x_{k}, y, t\right)>1-a+\varepsilon \text { or } N\left(x_{k}, y, t\right)<a-\varepsilon\right\} \in I .
\end{aligned}
$$

Conversely if (1.5) and (1.6) hold for every $\varepsilon>0$ and $t>0$, then $a=I_{(M, N)}-\lim \inf x$.

\section{Main results}

Definition 2.1. Let $(X, M, N, *, \diamond)$ be an IFMS and $\left(E_{k}\right)$ be a sequence of closed subsets of $X$. Then, I-outer and I-inner limits with respect to intuitionistic fuzzy metric $(M, N)$ are defined as follows:

$$
\mathrm{I}_{(M, N)}-\limsup _{\mathrm{k} \rightarrow \infty} \mathrm{E}_{\mathrm{k}}:=\left\{x \mid \forall \varepsilon>0, \exists \mathrm{K} \in \mathcal{Q}_{\mathrm{I}}^{\sharp}, \forall \mathrm{k} \in \mathrm{K}: \mathrm{E}_{\mathrm{k}} \cap \mathcal{B}_{x}(\varepsilon, \mathrm{t}) \neq \phi\right\}
$$

and

$$
\mathrm{I}_{(M, N)}-\liminf _{\mathrm{k} \rightarrow \infty} \mathrm{E}_{\mathrm{k}}:=\left\{x \mid \forall \varepsilon>0, \exists K \in \mathcal{Q}_{\mathrm{I}}, \forall \mathrm{k} \in \mathrm{K}: \mathrm{E}_{\mathrm{k}} \cap \mathcal{B}_{x}(\varepsilon, \mathrm{t}) \neq \phi\right\}
$$

for all $t>0$ and $x \in X$. The $I_{(M, N)}$-limit exists for sequence of closed sets $\left(E_{k}\right)$ if

$$
I_{(M, N)}-\limsup _{k \rightarrow \infty} E_{k}=I_{(M, N)}-\liminf _{k \rightarrow \infty} E_{k} .
$$

In this case, we state the sequence of sets is Kuratowski I-convergent in intuitionistic fuzzy metric and write,

$$
I_{(M, N)}-\lim _{k \rightarrow \infty} \inf E_{k}=I_{(M, N)}-\lim _{k \rightarrow \infty} \sup E_{k}=I_{(M, N)}-\lim _{k \rightarrow \infty} E_{k} .
$$

Furthermore, it is obvious that $Q_{\mathrm{I}} \subset Q^{\sharp} \mathrm{I}$, then

$$
\mathrm{I}_{(M, N)}-\lim _{k \rightarrow \infty} \inf \mathrm{E}_{k} \subseteq \mathrm{I}_{(M, N)}-\lim _{k \rightarrow \infty} \sup \mathrm{E}_{k} .
$$

In fact, $I_{(M, N)}-\lim _{k \rightarrow \infty} E_{k}=E$ if and only if

$$
\mathrm{I}_{(M, N)}-\lim _{k \rightarrow \infty} \sup \mathrm{E}_{\mathrm{k}} \subseteq \mathrm{E} \subseteq \mathrm{I}_{(M, N)}-\lim _{\mathrm{k} \rightarrow \infty} \inf \mathrm{E}_{\mathrm{k}} .
$$

Remark 2.2. $\mathrm{I}_{(\mathrm{M}, \mathrm{N})}-\lim _{\mathrm{k} \rightarrow \infty} \mathrm{E}_{\mathrm{k}}=\mathrm{E}$ if and only if the subsequent conditions are fulfilled:

(i) for each $x \in E$ and for every $\varepsilon, t>0$,

$$
\left\{k \in \mathbb{N}: \mathcal{B}_{x}(\varepsilon, t) \cap E_{k} \neq \phi\right\} \in \mathcal{F}(\mathrm{I}) ;
$$

(ii) for each $x \in X \backslash E$ there exist $\varepsilon>0$ and $t>0$,

$$
\left\{k \in \mathbb{N}: \mathcal{B}_{x}(\varepsilon, t) \cap E_{k}=\phi\right\} \in \mathcal{F}(\mathrm{I}) .
$$

Example 2.3. There are some examples of ideals and analogous Kuratowski I-convergence in IFMS.

(1) Let $I_{f}=\{E \subset \mathbb{N}: E$ is finite $\}$. It is obvious that $I_{f}$ is a non-trivial admissible ideal and hence usual Kuratowski I-convergence coincides with the Kuratowski $\mathrm{I}_{f}$-convergence.

(2) Take $\mathrm{I}_{\delta}=\{\mathrm{E} \subset \mathbb{N}: \delta(\mathrm{E})=0\}$. Then $\mathrm{I}_{\delta}$ is a non-trivial admissible ideal. Certainly, Kuratowski statistical convergence coincides with the Kuratowski $\mathrm{I}_{\delta}$-convergence.

(3) Fix $I_{0}=\{\phi\}$. $I_{0}$ is minimal ideal in $\mathbb{N}$. Therefore, for a sequence of closed sets $\left(E_{k}\right)$, we obtain

$$
I_{0(M, N)}-\liminf _{k \rightarrow \infty} E_{k}=\bigcap_{k=1}^{\infty} E_{k} \text { and } I_{0(M, N)}-\limsup _{k \rightarrow \infty} E_{k}=\operatorname{cl}\left(\bigcup_{k=1}^{\infty} E_{k}\right),
$$

where the closure of the set $E$ in IFMS $X$ is denoted by $c l(E)$. A sequence of sets $\left(E_{k}\right)$ is constant set 
if and only if it is Kuratowski $\mathrm{I}_{0}$-convergent.

(4) Let $K \subseteq \mathbb{N}$ and $K \neq \mathbb{N}$. Set $I_{K}=2^{K}$. Then, it is clear that $I_{K}$ is a non-trivial ideal in $\mathbb{N}$ and we obtain,

$$
I_{K(M, N)}-\liminf _{k \rightarrow \infty} E_{k}=\bigcap_{k \in \mathbb{N} \backslash K} E_{k} \text { and } I_{K(M, N)}-\limsup _{k \rightarrow \infty} E_{k}=\operatorname{cl}\left(\bigcup_{k \in \mathbb{N} \backslash K} E_{k}\right) .
$$

There is a closed set $E$ of $X$ in such a way that $E_{k}=E$ for each $k \in \mathbb{N} \backslash K$ if and only if it is Kuratowaski $\mathrm{I}_{\mathrm{K}}$-convergent.

Theorem 2.4. Let $(X, M, N, *, \diamond)$ be an IFMS and $\left(E_{k}\right)$ be a sequence of closed subsets of $X$. Then,

$$
I_{(M, N)}-\liminf _{k \rightarrow \infty} E_{k}=\bigcap_{K \in Q_{I}^{\sharp}} c l \bigcup_{k \in K} E_{k} \text { and } I_{(M, N)}-\underset{k \rightarrow \infty}{\limsup } E_{k}=\bigcap_{K \in Q_{I}} c l \bigcup_{k \in K} E_{k} .
$$

Proof. Let $K \in Q_{I}^{\sharp}$ be an arbitrary and $x \in I_{(M, N)}-\liminf _{k \rightarrow \infty} E_{k}$. Then, for all $t>0$ and for every $\varepsilon>0$, there exists $K_{1} \in Q_{\text {I }}$ such that for every $k \in K_{1}$, we have

$$
\mathrm{E}_{\mathrm{k}} \cap \mathcal{B}_{\mathrm{x}}(\varepsilon, \mathrm{t}) \neq \phi
$$

Using Lemma 1.4, we obtain $\mathrm{K} \cap \mathrm{K}_{1} \notin \mathrm{I}$. Consequently, there exists $\mathrm{k}_{0} \in \mathrm{K} \cap \mathrm{K}_{1}$ in such a way that $\mathrm{E}_{\mathrm{k}_{0}} \cap \mathcal{B}_{\mathrm{x}}(\varepsilon, \mathrm{t}) \neq \phi$. Thus,

$$
\left(\bigcup_{k \in K} E_{k}\right) \cap \mathcal{B}_{x}(\varepsilon, t) \neq \phi,
$$

which implies $x \in \mathrm{cl} \bigcup_{k \in K} E_{k}$ and holds for any $K \in Q_{I}^{\sharp}$. Therefore, $x \in \bigcap_{K \in Q_{I}^{\sharp}} c l \bigcup_{k \in K} E_{k}$.

On contrary, let $x \notin I_{(M, N)}-\lim _{k \rightarrow \infty} \inf E_{k}$, there exist $\varepsilon>0$ and for all $t>0$ in such a way that

$$
\mathrm{K}=\left\{\mathrm{k} \in \mathbb{N}: \mathrm{E}_{\mathrm{k}} \cap \mathcal{B}_{x}(\varepsilon, \mathrm{t})=\phi\right\} \notin \mathrm{I},
$$

i.e., $K \in Q_{I}^{\sharp}$. Thus

$$
\left(\bigcup_{k \in K} E_{k}\right) \cap \mathcal{B}_{x}(\varepsilon, t)=\phi
$$

This implies that $x \notin \mathrm{cl} \bigcup_{k \in K} E_{k}$. Consequently, $x \notin \bigcap_{K \in Q_{I}^{\sharp}} c l \bigcup_{k \in K} E_{k}$. This shows that

$$
I_{(M, N)}-\liminf _{k \rightarrow \infty} E_{k}=\bigcap_{K \in \mathcal{Q}_{I}^{\sharp}} \mathrm{cl} \bigcup_{k \in K} E .
$$

On the similar manner, one can prove that

$$
I_{(M, N)}-\limsup _{k \rightarrow \infty} E_{k}=\bigcap_{K \in Q_{I}} c l \bigcup_{k \in K} E_{k} .
$$

As a sequel of Theorem 2.4, for any given sequence the sets $\left(E_{k}\right), I_{(M, N)}-\limsup _{k \rightarrow \infty} E_{k}$ and $I_{(M, N)}-$ $\liminf _{k \rightarrow \infty} E_{k}$ are closed. 
Theorem 2.5. Let $(\mathrm{X}, \mathrm{M}, \mathrm{N}, *, \diamond)$ be an IFMS and $\left(\mathrm{E}_{\mathrm{k}}\right)$ be a sequence of closed subsets of $\mathrm{X}$. Then,

$$
\begin{aligned}
I_{(M, N)}-\liminf _{k \rightarrow \infty} E_{k} & =\left\{x \mid \text { for all } t>0, I_{(M, N)}-\lim _{k \rightarrow \infty} M\left(x, E_{k}, t\right)=1 \text { and } I_{(M, N)}-\lim _{k \rightarrow \infty} N\left(x, E_{k}, t\right)=0\right\}, \\
I_{(M, N)}-\limsup _{k \rightarrow \infty} E_{k} & =\left\{x \mid \text { for all } t>0, I_{(M, N)}-\limsup _{k \rightarrow \infty} M\left(x, E_{k}, t\right)=1 \text { and } I_{(M, N)}-\liminf _{k \rightarrow \infty} N\left(x, E_{k}, t\right)=0\right\} .
\end{aligned}
$$

Proof. For any closed set $\mathrm{E}$, we have

$$
M(x, E, t) \geqslant 1-\varepsilon \text { or } N(x, E, t) \leqslant \varepsilon \text { if and only if } E \cap \mathcal{B}_{x}(\varepsilon, t)=\phi .
$$

Let $I_{(M, N)}-\lim _{k \rightarrow \infty} M\left(x, E_{k}, t\right)=1$ and $I_{(M, N)}-\lim _{k \rightarrow \infty} N\left(x, E_{k}, t\right)=0$. Then, for all $t>0$ and for every $\varepsilon>0$, we have

$$
\left\{k \in \mathbb{N}: M\left(x, E_{k}, t\right) \geqslant 1-\varepsilon \text { or } N\left(x, E_{k}, t\right) \leqslant \varepsilon\right\} \in I .
$$

By equation (2.1), for all $t>0$ and for every $\varepsilon>0$, we obtain

$$
\left\{k \in \mathbb{N}: E_{k} \cap \mathcal{B}_{x}(\varepsilon, t)=\phi\right\} \in I .
$$

This implies that

$$
\left\{k \in \mathbb{N}: E_{k} \cap \mathcal{B}_{x}(\varepsilon, t) \neq \phi\right\} \in \mathcal{F}(\mathrm{I}) .
$$

Hence, $x \in I_{(M, N)}-\lim _{k \rightarrow \infty} \inf E_{k}$.

On the contrary, suppose $x \in I_{(M, N)}-\lim _{k \rightarrow \infty} \inf E_{k}$. For every $\varepsilon>0$ and $t>0$, there exists $K \in Q_{I}$ in such a way that $E_{k} \cap \mathcal{B}_{x}(\varepsilon, t) \neq \phi$ for every $k \in K$. Since

$$
\left\{k \in \mathbb{N}: E_{k} \cap \mathcal{B}_{x}(\varepsilon, t)=\phi\right\} \subseteq \mathbb{N} \backslash K,
$$

so

$$
\left\{k \in \mathbb{N}: E_{k} \cap \mathcal{B}_{x}(\varepsilon, t)=\phi\right\} \in I .
$$

By using equation (2.1), we obtain

$$
\left\{k \in \mathbb{N}: M\left(x, E_{k}, t\right) \geqslant 1-\varepsilon \text { or } N\left(x, E_{n}, t\right) \leqslant \varepsilon\right\} \in I .
$$

Therefore, $I_{(M, N)}-\lim _{k \rightarrow \infty} M\left(x, E_{k}, t\right)=1$ and $I_{(M, N)}-\lim _{k \rightarrow \infty} N\left(x, E_{k}, t\right)=0$. In a similar manner, for any closed set $\mathrm{E}$, we have

$$
M(x, E, t)<1-\varepsilon \text { or } N(x, E, t)>\varepsilon \text { if and only if } E \cap \mathcal{B}_{x}(\varepsilon, t) \neq \phi .
$$

Let $I_{(M, N)}-\lim _{k \rightarrow \infty} \inf M\left(x, E_{k}, t\right)=1$ and $I_{(M, N)}-\lim _{k \rightarrow \infty} \inf N\left(x, E_{k}, t\right)=0$. Then, for every $\varepsilon>0$ and $t>0$,

$$
\left\{k \in \mathbb{N}: M\left(x, E_{k}, t\right)<1-\varepsilon \text { or } N\left(x, E_{k}, t\right)>\varepsilon\right\} \notin I .
$$

Therefore, for every $\varepsilon, t>0$ and using inequality (2.2), we obtain

$$
\left\{k \in \mathbb{N}: E_{k} \cap \mathcal{B}_{x}(\varepsilon, t) \neq \phi\right\} \notin I .
$$

This implies that $x \in I_{(M, N)}-\lim _{k \rightarrow \infty} \sup E_{k}$.

On contrary, suppose $x \in I_{(M, N)}^{k \rightarrow \infty}-\lim _{k \rightarrow \infty} \sup E_{k}$. Then, for every $\varepsilon>0$ and $t>0$,

$$
\left\{k \in \mathbb{N}: E_{k} \cap \mathcal{B}_{x}(\varepsilon, t) \neq \phi\right\} \notin \mathrm{I} .
$$

Using Proposition 1.14 and inequality (2.2), we obtain $I_{(M, N)}-\lim _{k \rightarrow \infty} \sup M\left(x, E_{k}, t\right)=1$ and $I_{(M, N)}-$ $\lim _{k \rightarrow \infty} \inf N\left(x, E_{k}, t\right)=0$. 
Theorem 2.6. Let $(X, M, N, *, \diamond)$ be an IFMS and $\left(E_{k}\right)$ be a sequence of closed subsets of $X$. Then, for all $t>0$,

$$
\mathrm{I}_{(M, N)}-\lim _{k \rightarrow \infty} \inf E_{k}=\left\{x \mid \forall k \in \mathbb{N}, \exists y_{k} \in E_{k}: I_{(M, N)}-\lim _{k \rightarrow \infty} y_{k}=x\right\} .
$$

Proof. Assume that $x \in I_{(M, N)}-\lim _{k \rightarrow \infty} \inf E_{k}$. Therefore, by Theorem 2.5, $I_{(M, N)}-\lim _{k \rightarrow \infty} M\left(x, E_{k}, t\right)=1$ and $I_{(M, N)}-\lim _{k \rightarrow \infty} N\left(x, E_{k}, t\right)=0$. Now, for all $t>0$ and for every $\varepsilon>0$, we have

$$
\left\{k \in \mathbb{N}: M\left(x, E_{k}, t\right) \geqslant 1-\frac{\varepsilon}{2} \text { or } N\left(x, E_{k}, t\right) \leqslant \frac{\varepsilon}{2}\right\} \in I .
$$

Since the sequence $\left(E_{k}\right)$ is closed, for all $k \in \mathbb{N}$, there exists $\left(y_{k}\right) \in E_{k}$ such that

$$
M\left(x, y_{k}, t\right) \leqslant 2 M\left(x, E_{k}, t\right) \text { and } N\left(x, y_{k}, t\right) \geqslant 2 N\left(x, E_{k}, t\right)
$$

Now, for any sequence $y=\left(y_{k}\right)$ with $\left(y_{k}\right) \in E_{k}$ for all $k$, we have

$$
I_{(M, N)}-\lim _{k \rightarrow \infty} y_{k}=x .
$$

Conversely, suppose that $x$ belongs to the right hand side set of the equality (2.3). Then, there exists a sequence $y=\left(y_{k}\right)$ with $\left(y_{k}\right) \in E_{k}$ for all $k$ such that

$$
I_{(M, N)}-\lim _{k \rightarrow \infty} y_{k}=x .
$$

Then, for all $t>0$ and for every $\varepsilon$, we have

$$
\left\{k \in \mathbb{N}: M\left(x, E_{k}, t\right) \geqslant 1-\varepsilon \text { or } N\left(x, E_{k}, t\right) \leqslant \varepsilon\right\} \in I .
$$

The inequalities $M\left(x, y_{k}, t\right) \geqslant M\left(x, E_{k}, t\right)$ and $N\left(x, y_{k}, t\right) \leqslant N\left(x, E_{k}, t\right)$ imply the next inclusions

$$
\left\{k \in \mathbb{N}: M\left(x, E_{k}, t\right) \geqslant 1-\varepsilon \text { or } N\left(x, E_{k}, t\right) \leqslant \varepsilon\right\} \subseteq\left\{k \in \mathbb{N}: M\left(x, y_{k}, t\right) \geqslant 1-\varepsilon \text { or } N\left(x, y_{k}, t\right) \leqslant \varepsilon\right\} .
$$

So,

$$
\left\{k \in \mathbb{N}: M\left(x, E_{k}, t\right) \geqslant 1-\varepsilon \text { or } N\left(x, E_{k}, t\right) \leqslant \varepsilon\right\} \in I .
$$

This means that $I_{(M, N)}-\lim _{k \rightarrow \infty} M\left(x, E_{k}, t\right)=1$ and $I_{(M, N)}-\lim _{k \rightarrow \infty} N\left(x, E_{k}, t\right)=0$. By Theorem 2.5, we have $x \in \mathrm{I}_{(M, N)}-\lim _{k \rightarrow \infty} \inf \mathrm{E}_{\mathrm{k}}$.

Theorem 2.7. Let $(X, M, N, *, \diamond)$ be an IFMS and $\left(E_{k}\right)$ be a sequence of closed subsets of $X$. Then for every $t>0$,

$$
\mathrm{I}_{(\mathrm{M}, \mathrm{N})}-\lim _{\mathrm{k} \rightarrow \infty} \sup _{\mathrm{k}}=\left\{x \mid \exists \mathrm{K} \in \mathrm{Q}_{\mathrm{I}}^{\sharp}, \forall \mathrm{k} \in \mathrm{K}, \exists \mathrm{y}_{\mathrm{k}} \in \mathrm{E}_{\mathrm{k}}: x \in \mathrm{I}\left(\Gamma_{\mathrm{y}}\right)\right\},
$$

where $\mathrm{I}\left(\Gamma_{\mathrm{y}}\right)$ denotes the set of all I-cluster points of a sequence $\mathrm{y}$.

Proof. Suppose that $x \in I_{(M, N)}-\lim _{k \rightarrow \infty} \sup E_{k}$ be arbitrary. By Theorem 2.5,

$$
I_{(M, N)}-\limsup _{k \rightarrow \infty} M\left(x, E_{k}, t\right)=1 \text { and } I_{(M, N)}-\liminf _{k \rightarrow \infty} N\left(x, E_{k}, t\right)=0 .
$$

Using Propositions 1.13 and 1.14 , for all $t>0$ and for every $\varepsilon>0$, we have

$$
\left\{k \in \mathbb{N}: M\left(x, E_{k}, t\right)>1-\frac{\varepsilon}{2} \text { and } N\left(x, E_{k}, t\right)<\frac{\varepsilon}{2}\right\} \notin I .
$$

Since $E_{k}$ is closed, for all $k \in \mathbb{N}$, there exists $\left(y_{k}\right) \in E_{k}$ such that

$$
M\left(x, y_{k}, t\right) \leqslant 2 M\left(x, E_{k}, t\right) \text { and } N\left(x, y_{k}, t\right) \geqslant 2 N\left(x, E_{k}, t\right) .
$$


Now, we define a sequence $y=\left(y_{k}\right)$ with $\left(y_{k}\right) \in E_{k}$ for all $k$. Then

$$
\left\{k \in \mathbb{N}: M\left(x, y_{k}, t\right)>1-\varepsilon \text { and } N\left(x, y_{k}, t\right)<\varepsilon\right\} \notin I .
$$

Hence, $x \in \mathrm{I}\left(\Gamma_{\mathrm{y}}\right)$.

Conversely, suppose that $x$ belongs to the right hand side set of the equality (2.4). Then, there exists $K \in Q^{\sharp}$, a sequence $y=\left(y_{k}\right)$ with $\left(y_{k}\right) \in E_{k}$ for all $k \in K$ such that $x \in I\left(\Gamma_{y}\right)$. Then, for every $\varepsilon>0$ and $t>0$, we have

$$
\left\{k \in \mathbb{N}: M\left(x, y_{k}, t\right)>1-\varepsilon \text { and } N\left(x, y_{k}, t\right)<\varepsilon\right\} \notin I .
$$

Using the equations (1.1) and (1.2), we yield the following inclusion

$$
\left\{k \in \mathbb{N}: M\left(x, y_{n}, t\right)>1-\varepsilon \text { and } N\left(x, y_{n}, t\right)>\varepsilon\right\} \subseteq\left\{k \in \mathbb{N}: M\left(x, E_{k}, t\right)>1-\varepsilon \text { and } N\left(x, E_{k}, t\right)<\varepsilon\right\} .
$$

So, $K^{\prime}=\left\{k \in K: M\left(x, E_{k}, t\right)>1-\varepsilon\right.$ and $\left.N\left(x, E_{k}, t\right)<\varepsilon\right\} \notin I$. That is, $K^{\prime} \in Q_{I}^{\sharp}$. By equation (2.2), for every $k \in K^{\prime}$, we have $E_{k} \cap \mathcal{B}_{x}(\varepsilon, t) \neq \phi$. This implies that $x \in I_{(M, N)}-\lim _{k \rightarrow \infty} \sup E_{k}$.

Theorem 2.8. Let $(\mathrm{X}, \mathrm{M}, \mathrm{N}, *, \diamond)$ be an IFMS. Let sequence of closed subsets $\left(\mathrm{E}_{\mathrm{k}}\right)$ of $\mathrm{X}$ is I-monotonic increasing. Then, $\mathrm{I}_{(\mathrm{M}, \mathrm{N})}-\lim _{\mathrm{k} \rightarrow \infty} \mathrm{E}_{\mathrm{k}}$ exists and

$$
I_{(M, N)}-\lim _{k \rightarrow \infty} E_{k}=c l \bigcup_{i \in \mathbb{N}} E_{k_{i}} .
$$

Proof. Let $\left(E_{k}\right)$ be I-monotonic increasing sequence of closed subsets of $X$ and $c l \bigcup_{i \in \mathbb{N}} E_{k_{i}}=E$. It is obvious that, $E_{k_{i}} \subseteq E$ for every $i \in \mathbb{N}$. If $E=\phi$, then, for every $i \in \mathbb{N}$, we have $I_{(M, N)}-\lim _{k \rightarrow \infty} E_{k}=\phi$. Suppose that $E \neq \phi$ and $x \in \operatorname{cl} \bigcup_{i \in \mathbb{N}} E_{k_{i}}$. In this condition, for every $\varepsilon>0$ and $t>0$, we have

$$
\mathcal{B}_{x}(\varepsilon, t) \cap \bigcup_{i \in \mathbb{N}} E_{k_{i}} \neq \phi .
$$

Therefore, there exists $i_{0} \in \mathbb{N}$ in such a way that $\mathcal{B}_{x}(\varepsilon, t) \cap E_{k_{i_{0}}} \neq \phi$. Since sequence is increasing, i.e., $E_{k_{i_{0}}} \subseteq E_{k_{i}}, \forall i \geqslant i_{0}$. Define the set $P=\left\{p: p=k_{i}, i \geqslant i_{0}, k \in \mathbb{N}\right\}$. Thus $P \in \mathcal{F}(I)$ and

$$
\mathcal{B}_{x}(\varepsilon, t) \cap E_{p} \neq \phi, \forall p \in P \text {. }
$$

Therefore, we have $x \in I-\liminf _{k \rightarrow \infty} E_{k}$.

Now, we have to show that $I-\lim _{k \rightarrow \infty} \sup E_{k} \subseteq E$. Suppose that $x \in I-\lim _{k \rightarrow \infty} \sup E_{k}$. Then for all $t>0$ and every $\varepsilon>0$, there exists $K \in Q_{I}^{\sharp}$ in such a way that for every $k \in K$ we obtain

$$
\mathcal{B}_{x}(\varepsilon, t) \cap E_{k} \neq \phi .
$$

Since $\mathrm{K}_{1} \in \mathcal{F}(\mathrm{I})$ and $\mathrm{K} \notin \mathrm{I}$, using Lemma 1.4, we obtain $\mathrm{K} \cap \mathrm{K}_{1} \notin \mathrm{I}$. Then, there exists $\mathrm{k}_{\mathrm{i}_{0}} \in \mathrm{K} \cap \mathrm{K}_{1}$ such that

$$
\mathcal{B}_{\chi}(\varepsilon, t) \cap E_{k_{i_{0}}} \neq \phi \text {. }
$$

Hence, we have

$$
\mathcal{B}_{x}(\varepsilon, t) \cap \bigcup_{i \in \mathbb{N}} E_{k_{i}} \neq \phi .
$$

This implies that $x \in \mathrm{cl} \bigcup_{i \in \mathbb{N}} \mathrm{E}_{\mathrm{k}_{i}} \neq \phi$. This completes the proof.

Theorem 2.9. Let $(X, M, N, *, \diamond)$ be an IFMS. Let $\left(E_{k}\right)$ be an I-monotonic decreasing sequence of closed subsets of $X$. Then, $\mathrm{I}_{(\mathrm{M}, \mathrm{N})}-\lim _{\mathrm{k} \rightarrow \infty} \mathrm{E}_{\mathrm{k}}$ exists and

$$
I_{(M, N)}-\lim _{k \rightarrow \infty} E_{k}=\bigcap_{i \in \mathbb{N}} E_{k_{i}}
$$


Proof. Suppose that $\bigcap_{i \in \mathbb{N}} E_{k_{i}}=E$. If $x \in E$, then $x \in E_{k_{i}} \forall i \in \mathbb{N}$. Define the set $P=\left\{p: p=k_{i}, i \geqslant i_{0}, k \in\right.$ $\mathbb{N}$ \}. Thus $P \in \mathcal{F}(\mathrm{I})$. For every $\varepsilon>0, t>0$ and $p \in P$, we obtain

$$
\mathcal{B}_{x}(\varepsilon, t) \cap E_{p} \neq \phi .
$$

This implies that $x \in I_{(M, N)}-\lim _{k \rightarrow \infty} \inf E_{k}$.

Now, we have to prove that $I_{(M, N)}-\lim _{k \rightarrow \infty} \sup E_{k} \subseteq E$. Suppose that $x \in I_{(M, N)}-\lim _{k \rightarrow \infty} \sup E_{k}$. Then, for all $t>0$ and every $\varepsilon>0$, there exists $K \notin I$ in such a way that for every $p \in K$, we obtain

$$
\mathcal{B}_{\chi}(\varepsilon, t) \cap E_{p} \neq \phi .
$$

Since $K$ is infinite and $I$ be an admissible ideal. Then, for every $i \in \mathbb{N}$ there exists $p \in K$ such that $k_{i} \leqslant p$. Since the sequence is decreasing, the inclusion $E_{k_{i}} \supseteq E_{p}$ holds and consequently

$$
\mathcal{B}_{x}(r, t) \cap E_{k_{i}} \neq \phi .
$$

This implies that $x \in \mathrm{clE}_{k_{i}}$. Since $E_{k_{i}}$ is closed, hence, $x \in \bigcap_{i \in \mathbb{N}} E_{k_{i}}$. This step concludes the proof.

\section{Acknowledgment}

The authors would like to record their gratitude to the reviewers for their careful reading and making some useful corrections which improved the presentation of the paper.

\section{References}

[1] K. T. Atanassov, Intuitionistic fuzzy sets, Fuzzy Sets and Systems, 20 (1986), 87-96. 1

[2] K. Demirci, I-limit superior and limit inferior, Math. Commun., 6 (2001), 165-172. 1

[3] H. Fast, Sur la convergence statistique, (French) Colloq. Math., 2 (1951), 241-244. 1

[4] J. A. Fridy, On statistical Convergence, Analysis, 5 (1985), 301-313. 1

[5] J. A. Fridy, On statistical limit points, Proc. Amer. Math. Soc., 11 (1993), 1187-1192. 1

[6] J. Fridy, C. Orhan, Statistical limit superior and limit inferior, Proc. Amer. Math. Soc., 25 (1997), 3627-3631. 1

[7] A. George, P. Veeramani, On some results in fuzzy metric spaces, Fuzzy Sets and Systems, 64 (1994), 395-399. 1

[8] A. George, P. Veeramani, On some results of analysis for fuzzy metric spaces, Fuzzy Sets and Systems, 90 (1997), 365-368. 1

[9] V. A. Khan, K. M. A. S. Alshlool, S. A. A. Abdullah, Spaces of ideal convergent sequences of bounded linear operators, Numer. Funct. Anal. Optim., 39 (2018), 1278-1290. 1

[10] V. A. Khan, R. K. A. Rababah, H. Fatima, Yasmeen, M. Ahmad, Intuitionistic fuzzy I-convergent sequence spaces defined by bounded linear operator, ICICI Express Letters, 12 (2018), 955-962. 1

[11] P. Kostyrko, T. Salat, W. Wilczynski, I-convergence, Real Anal. Exchange, 26 (2000/01), 669-686. 1, 1.1, 1.2, 1.3, 1.4

[12] C. Kuratowski, Topologie, Vol. I, Państwowe Wydawnictwo Naukowe, Warsaw, (1958). 1, 1

[13] M. Mursaleen, S. A. Mohiuddine, Statistical convergence of double sequences in intuitionistic fuzzy normed spaces, Chaos Solitons Fractals, 41 (2009), 2414-2421. 1

[14] M. Mursaleen, S. A. Mohiuddine, O. H. H. Edely, On the ideal convergence of double sequences in intuitionistic fuzzy normed spaces, Comput. Math. Appl., 59 (2010), 603-611. 1

[15] J. H. Park, Intuitionistic fuzzy metric spaces, Chaos Solitons Fractals, 22 (2004), 1039-1046. 1, 1.8, 1.9, 1.10

[16] R. Saadati, J. H. Park, On the intuitionistic fuzzy topological spaces, Chaos Solitons Fractals, 27 (2006), 331-344. 1, 1.12

[17] T. Šalát, B. C. Tripathy, M. Ziman, On some properties of I-convergence, Tatra Mt. Math. Publ., 28 (2004), $279-286.1$

[18] M. Sen, On I-limit superior and I-limit inferior of sequences in intuitionistic fuzzy normed spaces, Int. J. Comput. Appl., 85 (2014), 30-33. 1

[19] Ö. Talo, Y. Sever, On Kuratowski I-convergence of sequences of closed sets, Filomat, 31 (2017), 899-912. 1.5, 1, 1.7

[20] Ö. Talo, Y. Sever, F. Basar, On statistically convergent sequences of closed sets, Filomat, 30 (2016), 1497-1509. 1

[21] L. A. Zadeh, Fuzzy sets, Inf. Control, 8 (1965), 338-353. 1 